\title{
A Note on Topp-Leone Odd Log-Logistic Inverse Exponential Distribution
}

\author{
Salman Abbas ${ }^{1, \star}$, Fakhar Mustafa ${ }^{2}$, Syed Ali Taqi ${ }^{1}$, Selen Cakmakyapan ${ }^{3}$, Gamze Ozel $^{4}$ \\ ${ }^{1}$ Department of Statistics, COMSATS University Islamabad, Lahore Campus, Lahore, Pakistan \\ ${ }^{2}$ Department of Mathematics, COMSATS University Islamabad, Sahiwal Campus, Sahiwal, Pakistan \\ ${ }^{3}$ Department of Statistics, Istanbul Medeniyet University, İstanbul, Turkey \\ ${ }^{4}$ Department of Statistics, Hacettepe University, Ankara, Turkey
}

\section{ARTICLE INFO}

\section{Article History}

Received 10 Mar 2019

Accepted 16 Jan 2020

\section{Keywords}

Inverse exponential distribution Topp-Leone odd log-logistic family Hazard function

Residual and reversed residual life functions

Unimodal

\begin{abstract}
The inverse exponential distribution is widely used in the field of reliability. In this article, we present a generalization of the inverse exponential distribution in formation of Topp-Leone odd log-logistic inverse exponential distribution. We provide a comprehensive account of some mathematical properties of the Topp-Leone odd log-logistic inverse exponential distribution. The possible shapes of the corresponding probability density function and hazard function are obtained and graphical demonstration are presented. The distribution is found to be unimodal. The results for moment, moment-generating function, and probability-generating function are computed. The residual and reversed residual functions are also obtained. The proposed method of maximum likelihood is used for the estimation of model parameters. The performance of the parameters is investigated through simulation. The usefulness of the proposed model is illustrated by means of a real data set.
\end{abstract}

(c) 2020 The Authors. Published by Atlantis Press B.V. This is an open access article distributed under the CC BY-NC 4.0 license (http://creativecommons.org/licenses/by-nc/4.0/).

\section{INTRODUCTION}

Keller and Kamath [1] introduced the inverse exponential (IEx) distribution to study the reliability of computer control (CNC) machine tools. Lin et al. [2] discussed the IEx distribution in term of different causes of failure for the machines. They obtained the maximum likelihood estimator and confidence limits for the parameter and the reliability function using complete samples. They also compared this model with the inverted Gaussian and log-normal distributions based on a maintenance data set. Sanku Dey [3] and Gyan Prakash [4] studied the IEx distribution according to Bayesian point of view. Abouammoh and Alshingiti [5] worked on the addition of an extra shape parameter to obtain the generalized IEx distribution. It is to be noted that this distribution originated from the exponentiated Frechet distribution studied by Nadarajah and Kotz [6].

Several interesting generalizations of the IEx distribution have been derived including transmuted-generalized IEx distribution by Elbatal [7], the Kumaraswamy IEx distribution by Oguntunde et al. [8], the transmuted IEx distribution by Oguntunde and Adejumo [9], the exponentiated-generalized IEx distribution by Oguntunde et al. [10], and the beta-generalized IEx distribution by Bakoban and Abu-Zinadah [11].

The most common exhibition of hazard rates appeared in practice is the bathtub shaped. A simple model form bathtub-shaped hazard rates is the Topp-Leone distribution introduced by Topp and Leone [12]. The quantile function of this model has closed-form and the model is also very flexible to generate the data.

We start by defining the class of probability distribution introduced by Alizadeh et al. [13] using the cumulative distribution function (cdf) of the odd log-logistic (OLL) distribution as a generator and named as the Topp-Leone odd log-logistic (TLOLL-G) family of distributions. The proposed family has two additional shape parameters. The distribution function (cdf) of the proposed class of distributions is given as follows:

$$
F(x)=\left[1-\left(1-\frac{G(x ; \xi)^{a}}{G(x: \xi)^{a}+(x \xi)^{a}}\right)^{2}\right]^{b}
$$

${ }^{\star}$ Corresponding author. Email: salmanabbas@ciitwah.edu.pk 
and the corresponding density function (pdf) of the proposed family is given as

$$
f(x)=\frac{2 a b g(x ; \xi) G(x, \xi)^{a-1}(x ; \xi)^{2 a-1}}{\left[G(x ; \xi)^{a}+(x ; \xi)^{a}\right]^{3}}\left[1-\left(1-\frac{G(x ; \xi)^{a}}{G(x: \xi)^{a}+(x \xi)^{a}}\right)^{2}\right]^{b-1},
$$

where $a>0$ and $b>0$ are the shape parameters, $\xi$ is the vector parameter of the parent $G(x), g(x)$ is the derivative of $G(x)$, and $\overline{G(x)}=$ $1-G(x)$.

In this study, we introduce a generalization of the IEx distribution named as the Topp-Leone odd log-logistic inverse exponential (TLOLLIEx) distribution. The motivation of this generalization of IEx distribution is to enhance its applicability and adaptability. The addition of two shape parameters makes it more compatible for modeling the real life scenarios.

The organization of this article is as follows: The proposed TLOLL-IEx distribution and its properties are given in Section 2. Estimation of the model parameters is computed in Section 3. The performance of the parameters is investigated through simulation in Section 4. The application of the proposed model is illustrated in Section 5. The discussion ends with some concluding remarks in Section 6.

\section{TOLL-IEx DISTRIBUTION AND ITS PROPERTIES}

In this section, we derive the density function of the TLOLL-IEx distribution. For this aim, we consider the cdf of IEx distribution as

$$
G(x)=e^{-\frac{\lambda}{x}}, \quad x>0, \lambda>0
$$

and the pdf is given by

$$
g(x)=\frac{\lambda}{x^{2}} e^{-\frac{\lambda}{x}}, \quad x>0, \lambda>0
$$

Using (2.1) in (1.1), we have the cdf of TLOLL-IEx distribution as

$$
F(x)=\left[1-\left(1-\frac{e^{-\frac{\lambda}{x}}}{e^{-\frac{\lambda}{x}}+\left(1-e^{-\frac{\lambda}{x}}\right)^{a}}\right)^{2}\right]^{b}, x>0, a, b, \lambda>0 .
$$

The corresponding density of the TLOLL-IEx distribution is obtained by differentiating Eq. (2.1) and given as

$$
f(x)=\frac{2 a b \frac{\lambda}{x^{2}} e^{-\frac{a \lambda}{x}}\left(1-e^{-\frac{\lambda}{x}}\right)^{2 a-1}}{\left[e^{-\frac{a \lambda}{x}}+\left(1-e^{-\frac{\lambda}{x}}\right)^{a}\right]^{3}}\left[1-\left(1-\frac{e^{-\frac{\lambda}{x}}}{e^{-\frac{\lambda}{x}}+\left(1-e^{-\frac{\lambda}{x}}\right)^{a}}\right]^{2}\right]^{b-1}, x>0, a, b, \lambda>0 .
$$

We study some useful and important properties of the new distribution. The distributional properties of the proposed distribution is obtained for real values of $a$ and $b$.

\subsection{Shape}

The density function TLOLL-IEx distribution as form (2.1) is given as

$$
f(x)=\frac{2 a b \frac{\lambda}{x^{2}} e^{-\frac{a \lambda}{x}}\left(1-e^{-\frac{\lambda}{x}}\right)^{2 a-1}}{\left[e^{-\frac{a \lambda}{x}}+\left(1-e^{-\frac{\lambda}{x}}\right)^{a}\right]^{3}}\left[1-\left(1-\frac{e^{-\frac{\lambda}{x}}}{e^{-\frac{\lambda}{x}}+\left(1-e^{-\frac{\lambda}{x}}\right)^{a}}\right]^{2}\right]^{b-1} .
$$


For real $b$, we use following series representation given by Prudnikov et al. (1986) as

$$
(1+x)^{\alpha}=\sum_{j=0}^{\infty} \frac{\Gamma(\alpha+1)}{j !(\alpha+1-j)} c^{j}, \quad \alpha>0 .
$$

Then, the pdf of TLOLL-IEx distribution is written as

$$
f(x)=\sum_{k=0}^{\infty} d_{k+1} \gamma \lambda x^{-2}\left(e^{-\frac{\lambda}{x}}\right)^{\gamma} .
$$

Here, $d_{k}$ is given by

$$
d_{k}=\sum_{i=0}^{\infty} \sum_{j=0}^{2 i}(-1)^{i+j}\left(\begin{array}{c}
b \\
i
\end{array}\right)\left(\begin{array}{c}
2 i \\
j
\end{array}\right) c_{k, j} .
$$

We define $c_{k, j}$ as

$$
c_{k, j}=\frac{1}{b_{0, j}}\left(a_{k, j}-\frac{1}{b_{0, j}} \sum_{r=1}^{k} b_{k, j} c_{k-r, j}\right),
$$

where $b_{k, j}=h_{k}(a, j)$ and $a_{k, j}=\sum_{l=k}^{\infty}(-1)^{l+k}\left(\begin{array}{c}j a \\ l\end{array}\right)\left(\begin{array}{l}l \\ k\end{array}\right)$. Hence, in (2.5) $a$ and $b$ are real numbers. The density of TLOLL-IEx distribution is a weighted sum of infinite IEx distribution or it can also be termed specifically as non-central IEx distribution. Figure 1 demonstrates some of the possible shapes of the density function for the selected values of $a, b$, and $\lambda$.
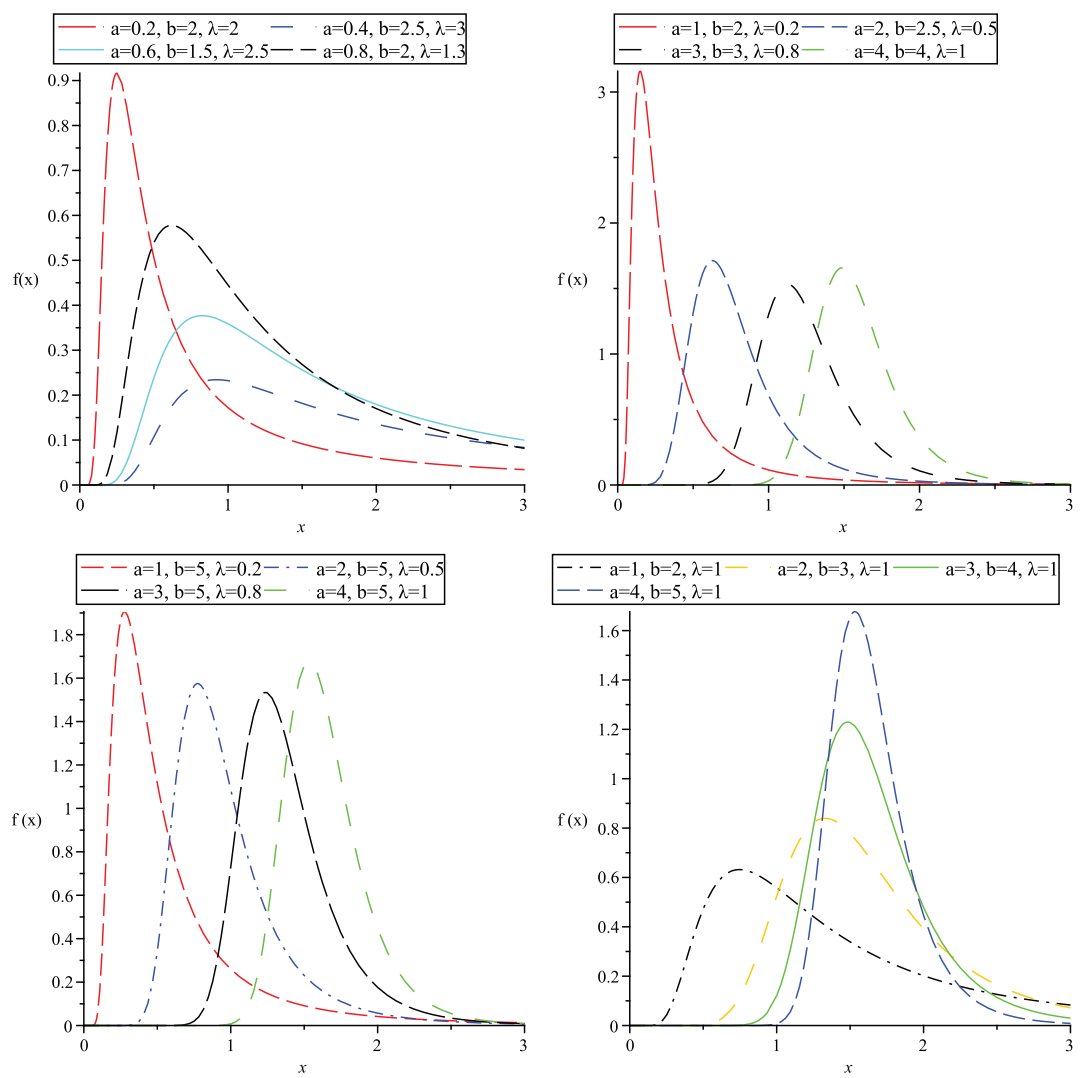

Figure 1 The density function for the Topp-Leone odd log-logistic inverse exponential (TLOLL-IEx) distribution for several values of parameters. 


\section{Quantile Function and Various Relevant Measures}

The quantile function of TLOLL-IE distribution corresponding to (2.1) is given by

$$
x=Q(u)=\frac{1}{\lambda}\left(\frac{\left[1-\left(1-u^{\frac{1}{b}}\right)^{-\frac{1}{2}}\right]^{\frac{1}{a}}}{1+\left[1-\left(1-u^{\frac{1}{b}}\right)^{-\frac{1}{2}}\right]^{\frac{1}{a}}}\right], 0<u<1 .
$$

The above expression concede us to extract the following form of statistical measures for the proposed model i. The first quartile Q1, the second quartile Q2(median), and the third quartile Q3 of the TLOLL-IE distribution correspond to the values $\mathrm{u}=0.25,0.50$, and 0.75 , respectively. ii. The skewness and kurtosis can be calculated by using the following relations, respectively. Bowleys skewness is based on quartiles in Kenney and Keeping (1962) calculated by

$$
s k 3=\frac{Q_{3}-2 Q_{2}+Q_{1}}{Q_{3}-Q_{1}} .
$$

Moors kurtosis (Moors, 1988) is based on octiles via the form

$$
s k 4=\frac{q\left(\frac{7}{8}\right)-q\left(\frac{5}{8}\right)-q\left(\frac{3}{8}\right)+q\left(\frac{1}{8}\right)}{q\left(\frac{6}{8}\right)-q\left(\frac{2}{8}\right)},
$$

where $q($.$) is the quantile function explain in (3.2).$

\section{Asymptotics}

Corollary 3.1: Let $c=\inf (x \mid G(x))>0$. Then, the asymptotics of $F(x), f(x)$ and $h(x)$ when $x \rightarrow c$ are, respectively, given as follows:

$$
\begin{array}{r}
F(x) \sim\left[2 a e^{-\frac{\lambda}{x}}\right]_{b}^{b} \text { as } x \longrightarrow c, \\
f(x) \sim b(2 a)^{\lambda} \frac{\lambda}{x^{2}}\left(e^{-\frac{\lambda}{x}}\right)^{b} \text { as } x \longrightarrow c, \\
h(x) \sim b(2 a)^{\lambda} \frac{\lambda}{x^{2}}\left(e^{-\frac{\lambda}{x}}\right)^{b} \text { as } x \longrightarrow c .
\end{array}
$$

Corollary 3.2: The asymptotics of $F(x), f(x)$ and $h(x)$ when $x \rightarrow \infty$ are, respectively, given by

$$
\begin{gathered}
1-F(x) \sim b\left(1-e^{-\frac{\lambda}{x}}\right)^{2 a} \text { as } x \longrightarrow 0, \\
f(x) \sim 2 a b \frac{\lambda}{x^{2}} e^{-\frac{\lambda}{x}}\left(1-e^{-\frac{\lambda}{x}}\right)^{2 a-1} \text { as } x \longrightarrow 0, \\
h(x) \sim \frac{2 a \frac{\lambda}{x^{2}} e^{-\frac{\lambda}{x}}}{1-e^{-\frac{\lambda}{x}}} \text { as } x \longrightarrow 0 .
\end{gathered}
$$

\subsection{Moment}

The $r$ th noncentral moment, $\mu_{r}^{\prime}=E\left(X^{r}\right)$ of the TLOLL family is given as

$$
\mu_{r}^{\prime}=E\left[x^{r}\right]=\sum_{k=0}^{\infty} d_{k+1} \int_{0}^{\infty} x^{r} \pi_{k+1}(x),
$$


where $\pi_{k+1}(x)=\gamma g(x) G(x)^{\gamma-1}$. After some calculation, the $r$ th moments of the TLLOL-IE distribution are derived as

$$
\mu_{r}^{\prime}=\sum_{k=0}^{\infty} d_{k+1}(\gamma \lambda)^{r} \Gamma(1-r)
$$

One can see, (2.6) is an infinite weighted sum of moments of the IEx distribution. The higher-order moments can be obtained by substituting $r<0$ in (2.6). The variance, skewness, and kurtosis measures can be calculated using the relations given by

$$
\begin{gathered}
\operatorname{Var}(x)=E\left(x^{2}\right)-[E(x)]^{2}, \\
\operatorname{Skewness}(x)=\frac{E\left(x^{3}\right)-3 E(x) E\left(x^{2}\right)+2 E^{3}(x)}{\operatorname{Var}^{\frac{3}{2}}(x)}, \\
\text { kurtosis }(x)=\frac{E\left(x^{4}\right)-4 E(x) E\left(x^{3}\right)+6 E\left(x^{2}\right) E^{2}(x)-E^{3}(x)}{\operatorname{Var}^{2}(x)} .
\end{gathered}
$$

\subsection{Moment-Generating Function and Probability-Generating Function}

The moment-generating function (mgf) of the TLOLL-IEx distribution is obtained by

$$
M_{x}(t)=\int_{-\infty}^{\infty} e^{t x} f(x)
$$

where $e^{t x}=\sum_{h=0}^{\infty} \frac{t^{x} x^{h}}{h !}$. Using the pdf (2.4), the mgf of the TLOLL-IEx distribution is given as follows:

$$
M_{x}(t)=\sum_{k, h=0}^{\infty} \frac{t^{m}}{h !} d_{k+1}(\gamma \lambda)^{1-h} \Gamma(1-h),
$$

Similarly, the probability-generating function (pgf) of the TLOLL-IEx distribution is defined as follows:

$$
\delta_{x}(t)=\int_{0}^{\infty} t^{x} \sum_{k=0}^{\infty} d_{k+1} \gamma \frac{\lambda}{x^{2}}\left(e^{-\frac{\lambda}{x}}\right)^{\gamma} d t
$$

where $t^{m}=\sum_{m=0}^{\infty} \frac{(\ln t)^{m} x^{m}}{m !}$, by solving the above expression, the pgf of the TLOLL-IEx distribution is given as follows:

$$
\rho_{x}(t)=\sum_{k, m=0}^{\infty} \frac{(\ln t)^{m}}{m !} d_{k+1}(\gamma \lambda)^{1-m} \Gamma(1-m) .
$$

Some reliability measure including hazard rate function also known as reliability function $r f$, residual life function, and reversed residual life function. The expression of survival function of the TLOLL-IEx distribution is given as follows:

$$
R(x)=1-\sum_{i, k=0}^{\infty} \sum_{j=0}^{2 i} d_{k}\left(e^{-\frac{\lambda}{x}}\right)^{\gamma}, x>0, a, b, \lambda>0
$$

\subsection{Hazard Function}

The hazard function for any probability distribution is given as

$$
h(x)=\frac{f(x)}{1-f(x)}
$$


For TLLOL-IE distribution, the hazard function is obtained by

$$
h(x)=\frac{\sum_{k=0}^{\infty} d_{k+1} \gamma \lambda x^{-2} e^{-\frac{\lambda}{x}}\left(e^{-\frac{\lambda}{x}}\right)^{\gamma-1}}{1-\sum_{i, k=0}^{\infty} \sum_{j=0}^{2 i} d_{k}\left(e^{-\frac{\lambda}{x}}\right)^{\gamma}}, x>0, a, b, \lambda>0
$$

Figure 2 presents, the plots of the hazard function for TLOLL-IEx distribution with several values of parameters. As seen in Figure 2, the hazard function of the TLOLL-IEx distribution is very flexible. The reversed hazard function is obtained as follows:

$$
r(x)=\frac{2 a b \frac{\lambda}{x^{2}} e^{-\frac{a \lambda}{x}}\left(1-e^{-\frac{\lambda}{x}}\right)^{2 a-1}}{\left[e^{-\frac{a \lambda}{x}}+\left(1-e^{-\frac{\lambda}{x}}\right)^{a}\right]^{3}\left(1-\left(1-\frac{e^{-\frac{\lambda}{x}}}{e^{-\frac{\lambda}{x}}+\left(1-e^{-\frac{\lambda}{x}}\right)^{a}}\right)^{2}\right)}
$$

and the cumulative hazard function is given by

$$
H(x)=-\log \left(1-\left[1-\left(1-\frac{e^{-\frac{\lambda}{x}}}{e^{-\frac{\lambda}{x}}+\left(1-e^{-\frac{\lambda}{x}}\right)^{a}}\right)^{2}\right]\right.
$$

\subsection{Moments for Residual and Reversed Residual Life}

The $s-t h$ moment of the residual life, says $m_{s}(t)=E\left[(x-y)^{n}|X>y|\right], n=1,2, \ldots$ is unusually obtained using cdf. The $s-t h$ moment of the residual life for $X$ is given by

$$
m_{s}(t)=\frac{1}{R(y)} \int_{y}^{\infty}(x-y)^{s} d F(x)
$$
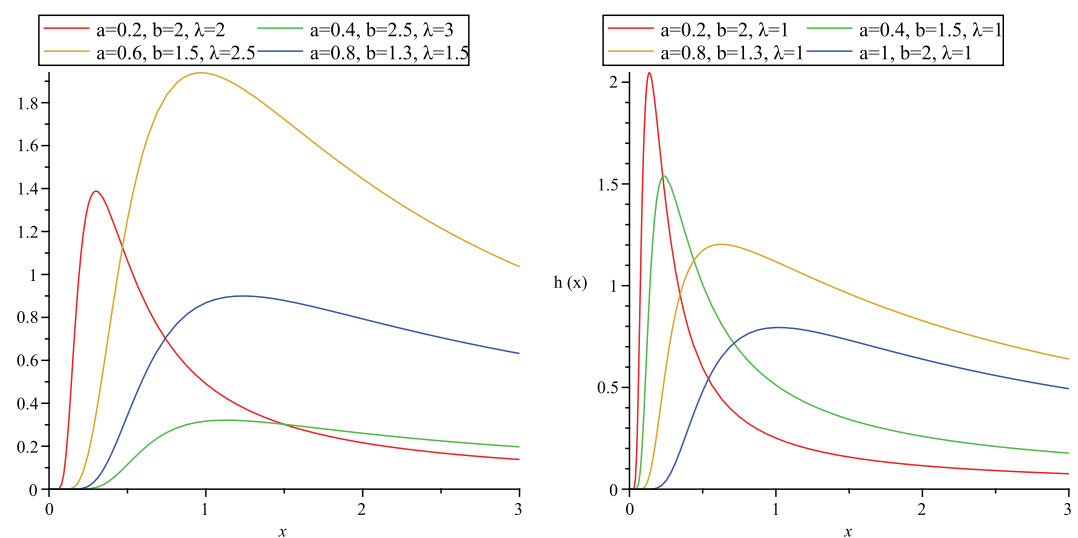

Figure 2 Plots for the hazard function of Topp-Leone odd log-logistic inverse exponential (TLOLL-IEx) distribution for several values of parameters. 
the final expression of the moment of the residual life is given by

$$
\begin{aligned}
m_{s}(y) & =\frac{1}{R(y)} \sum_{k, r=0}^{\infty}(-y)^{s-r} y_{k}\left(\begin{array}{l}
s \\
r
\end{array}\right) \int_{y}^{\infty} x^{r} \pi_{k+1}(x) \\
& =\frac{1}{R(y)} \sum_{k, r=0}^{\infty}(-y)^{s-r} y_{k}\left(\begin{array}{l}
s \\
r
\end{array}\right) d_{k+1} \gamma\left((1-r), \frac{\gamma \lambda}{y}\right) .
\end{aligned}
$$

The mean residual life of $X$ shows the expectation of additional life length for a unit which survive at age $y$. It can be easily computed by setting the value $s=1$. The $p-t h$ moment of the reversed residual life says $M_{p}(t)=E\left[(y-1)^{n}|X \leq y|\right]$, for $y>0$ and $p=1,2, \ldots$. is uniquely computed using $F(y)$ and given by

$$
M_{p}(y)=\frac{1}{F(y)} \int_{0}^{y}(y-x)^{n} d F(x)
$$

The $p-t h$ moment of the reversed residual life is determine as

$$
\begin{aligned}
M_{p}(Y) & =\frac{1}{F(y)} \sum_{k, r=0}^{\infty}(-1) y_{k}\left(\begin{array}{l}
n \\
r
\end{array}\right) t^{p-r} \int_{y}^{\infty} x^{r} \pi_{k+1}(x), \\
& =\frac{1}{F(y)} \sum_{k \cdot r=0}^{\infty}(-1) y_{k}\left(\begin{array}{c}
p \\
r
\end{array}\right) y^{p-r} d_{k+1} \gamma\left((1-r), \frac{\gamma \lambda}{y}\right) .
\end{aligned}
$$

\section{INFERENCE}

In this section, the method of maximum likelihood is considered for the estimation of parameters of TLOLL-IEx distribution. Let, $x_{1}, \ldots . ., x_{n}$ be the random sample from the TLOLL-IEx distribution with shape parameters $a, b$, and $p * 1$ is baseline vector parameter $\lambda$. The loglikelihood function for $\Theta=\left(a, b, \lambda^{T}\right)^{T}$, say $L=L(\Theta)$, is obtained by

$$
\begin{aligned}
L= & n \log (2)+n \log (a)+n \log (b)+\sum_{i=0}^{n} \frac{\lambda \log \left(e^{-\frac{\lambda}{x}}\right)}{x^{2}}+(a-1) \sum_{i=0}^{n} \log \left(e^{-\frac{\lambda}{x}}\right) \\
& +(2 a-1) \sum_{i=0}^{n} \log \left(1-e^{-\frac{\lambda}{x}}\right)-3 \sum_{i=0}^{n} \log \left(\left(e^{-\frac{\lambda}{x}}\right)^{a}+\left(1-e^{-\frac{\lambda}{x}}\right)^{a}\right) \\
& +(b-1) \sum_{i=1}^{n} \log \left(1-\left(1-\frac{\left.e^{-\frac{\lambda}{x}}\right)^{a}}{\left(e^{-\frac{\lambda}{x}}\right)^{a}+\left(1-e^{-\frac{\lambda}{x}}\right)^{a}}\right)^{2}\right)
\end{aligned}
$$

We can maximize the above equation using R (optim function), SAS (PROCNLMIXED), and Ox program (MaxBFGS sub-routine) or by solving the nonlinear likelihood equations by differentiation. The score vector components, say $I(\Theta)=\left(\frac{\partial L}{\partial a}, \frac{\partial L}{\partial b}, \frac{\partial L}{\partial \lambda}\right)^{T}=\left(I_{a}, I_{b}, I_{\lambda}^{T}\right)^{T}$, are given as follows:

$$
\begin{gathered}
I_{a}=\frac{n}{a}+\sum_{i=0}^{n} \log (w)+2 \sum_{i=0}^{n} \log (1-w)-3 \sum_{i=0}^{n} \frac{(w)^{a} \log (w)+(1-w)^{a} \log (1-w)}{(w)^{a}+(1-w)^{a}}+(b-1) \\
\times \sum_{i=1}^{n}-\frac{2\left(1-\frac{(w)^{a}}{(w)^{a}+(1-w)^{a}}\right)\left(\frac{(w)^{a}\left((w)^{a} \log (w)+(1-w)^{a} \log (1-w)\right)}{\left((w)^{a}+(1-w)^{a}\right)^{2}}-\frac{(w)^{a} \log (w)}{(w)^{a}+(1-w)^{a}}\right)}{1-\left(1-\frac{(w)^{a}}{(w)^{a}+(1-w)^{a}}\right)^{2}} \\
I_{b}=\frac{n}{b}+\sum_{i=1}^{n} \log \left(1-\left(1-\frac{(w)^{a}}{(w)^{a}+(1-w)^{a}}\right)^{2}\right)
\end{gathered}
$$




$$
\begin{aligned}
& I_{\lambda}=-3 \sum_{i=0}^{n} \frac{\frac{a w(1-w)^{a-1}}{x}-\frac{a(w)^{a}}{x}}{(w)^{a}+(1-w)^{a}}+(2 a-1) \sum_{i=0}^{n} \frac{w}{x(1-w)}-(a-1) \sum_{i=0}^{n} \frac{1}{x}-\frac{\lambda \sum_{i=0}^{n} \frac{1}{x}}{x^{2}}+\frac{\sum_{i=0}^{n} \log (w)}{x^{2}}+(b-1) \\
& \quad \times \sum_{i=1}^{n}-\frac{2\left(1-\frac{(w)^{a}}{(w)^{a}+(1-w)^{a}}\right)\left(\frac{\left(\frac{a w(1-w)^{a-1}}{x}-\frac{a(w)^{a}}{x}\right)(w)^{a}}{\left((w)^{a}+(1-w)^{a}\right)^{2}}+\frac{a(w)^{a}}{x\left((w)^{a}+(1-w)^{a}\right)}\right)}{1-\left(1-\frac{(w)^{a}}{(w)^{a}+(1-w)^{a}}\right)^{2}} .
\end{aligned}
$$

Here, $w=e^{-\frac{\lambda}{x}}$. We can find the maximum likelihood estimations (MLEs) by setting the above equations equal to 0 and solving them iteratively. The Fisher information matrix for the parameters of the TLOLL-IEx distribution is obtained by

$$
\begin{gathered}
\left(\begin{array}{l}
\hat{a} \\
\hat{b} \\
\hat{\lambda}
\end{array}\right) \sim N\left(\left(\begin{array}{l}
a \\
b \\
\lambda
\end{array}\right),\left(\begin{array}{lll}
\hat{J}_{a a} & \hat{J}_{a b} & \hat{J}_{a \lambda} \\
& \hat{J}_{b b} & \hat{J}_{b \lambda} \\
& & \hat{J}_{\lambda \lambda}
\end{array}\right)\right) \\
\\
\\
\frac{1}{J}=-E\left(\begin{array}{ccc}
J_{a a} & J_{a b} & J_{a \lambda} \\
& J_{b b} & J_{b \lambda} \\
& & J_{\lambda \lambda}
\end{array}\right) .
\end{gathered}
$$

By determining the inverse dispersion matrix, the asymptotic variances and covariances of the MLEs for $a, b$, and $\lambda$ can be obtained. Using above equations, approximate $100(1-\lambda) \%$ confidence intervals for $a, b$, and $\lambda$ are, respectively, obtained as

$$
\hat{a} \pm Z_{\frac{\gamma}{2}} \sqrt{a a}, \hat{b} \pm Z_{\frac{\gamma}{2}} \sqrt{b b}, \hat{\lambda} \pm Z_{\frac{\gamma}{2}} \sqrt{\lambda \lambda},
$$

where $Z_{\gamma}$ is the upper $100_{\gamma \text { th }}$ quantile of the standard normal distribution.

\section{SIMULATION STUDY}

In this section, we conduct a simulation study. We generate 10,000 samples of size, $n=50,100,250,500$ and $n=1000$ of the TLOLL-IEx model. The evaluation of estimates is based on the mean, the mean squared error (MSE), and We use R software for computation. The results in Table 1 show that the estimates are closer to the true values of the parameters from all sample size which clearly indicates that estimate are quite suitable. As the value of $n$ increases, we can see that the estimates tend to move toward their true values which justifies the fact of asymptotic normality.

\section{APPLICATION}

In this part, we discuss the applicability of TLOLL-IEx model in real-life phenomena. We consider the data of the tensile strength, measured in GPa, of 69 carbon fibers tested under tension at gauge lengths of $20 \mathrm{~mm}$ given by Chwastiak et al. [14 ]. The data is given in Table 2 . Then, fitted distributions and their abbreviations are presented in Table 3.

The unknown parameters of each distribution are estimated using the maximum likelihood method. AIC (Akaike Information Criterion), CAIC (Consistent Akaike Information Criterion), and BIC (Bayesian Information Criterion) are used as goodness of fit measure. The estimated parameters of the fitted models, AIC, CAIC, and BIC are presented in Table 4 . The values in this table clearly justify that the TLOLLIEx distribution provides better fits than other models.

We also obtain a visual comment with histogram given in Figure 3 for the best model.

\section{CONCLUSION}

In this paper, the discussion has been carried out through the generalization of the IEx distribution. We motivate from the TLOLL family of distributions and named the proposed model as TLOLL-IEx distribution. Several structural characteristics of the proposed distribution are derived and discussed. The maximum likelihood method is employed for estimating the model parameters. For effectiveness of the derived model, we consider a real-life data set and the results are compared with some well-known existing distributions. The TLOLL-IEx distribution is found unimodal. We hope that this generalization will applicable in the fields of reliability and lifetime analysis. 
Table 1 Estimated biases, mean squared errors (MSEs), average lenghts(ALS) for the several parameter values.

\begin{tabular}{|c|c|c|c|c|c|c|c|c|c|c|c|}
\hline \multirow[b]{2}{*}{$\lambda$} & \multirow{2}{*}{$\begin{array}{c}b=1 \\
a\end{array}$} & \multirow[b]{2}{*}{$n$} & \multicolumn{3}{|c|}{ Mean } & \multicolumn{3}{|c|}{ MSE } & \multicolumn{3}{|c|}{$\mathbf{A L}$} \\
\hline & & & $\lambda$ & $b$ & $a$ & $\lambda$ & $b$ & $a$ & $\lambda$ & $b$ & $a$ \\
\hline \multirow{5}{*}{0.5} & \multirow{5}{*}{0.5} & 50 & 0.661 & 1.321 & 0.783 & 0.312 & 0.215 & 1.315 & 0.831 & 0.737 & 0.749 \\
\hline & & 100 & 0.601 & 1.135 & 0.632 & 0.234 & 0.177 & 1.103 & 0.557 & 0.811 & 0.861 \\
\hline & & 250 & 0.549 & 1.075 & 0.554 & 0.133 & 0.121 & 0.311 & 0.313 & 0.853 & 0.882 \\
\hline & & 500 & 0.531 & 1.041 & 0.516 & 0.031 & 0.037 & 0.108 & 0.104 & 0.873 & 0.931 \\
\hline & & 1000 & 0.503 & 1.009 & 0.505 & 0.011 & 0.015 & 0.036 & 0.023 & 0.941 & 0.953 \\
\hline \multirow{5}{*}{0.5} & \multirow{5}{*}{2} & 50 & 0.537 & 1.319 & 2.417 & 0.578 & 0.315 & 2.112 & 1.731 & 0.821 & 0.886 \\
\hline & & 100 & 0.522 & 1.217 & 2.313 & 0.441 & 0.257 & 1.011 & 1.335 & 0.851 & 0.922 \\
\hline & & 250 & 0.519 & 1.033 & 2.139 & 0.361 & 0.113 & 0.713 & 0.716 & 0.885 & 0.936 \\
\hline & & 500 & 0.509 & 1.015 & 2.101 & 0.131 & 0.057 & 0.317 & 0.318 & 0.911 & 0.948 \\
\hline & & 1000 & 0.501 & 1.003 & 2.011 & 0.053 & 0.013 & 0.103 & 0.107 & 0.942 & 0.953 \\
\hline \multirow{5}{*}{3} & \multirow{5}{*}{2.5} & 50 & 0.631 & 1.353 & 1.619 & 0.485 & 0.553 & 0.972 & 2.538 & 0.831 & 0.841 \\
\hline & & 100 & 0.539 & 1.172 & 1.761 & 0.349 & 0.331 & 0.609 & 1.549 & 0.866 & 0.883 \\
\hline & & 250 & 0.526 & 1.105 & 1.837 & 0.111 & 0.145 & 0.363 & 0.649 & 0.913 & 0.914 \\
\hline & & 500 & 0.513 & 1.051 & 1.935 & 0.018 & 0.051 & 0.131 & 0.341 & 0.937 & 0.938 \\
\hline & & 1000 & 0.501 & 1.011 & 1.992 & 0.001 & 0.012 & 0.015 & 0.131 & 0.944 & 0.948 \\
\hline
\end{tabular}

Table $2 \mid$ Carbon fiber data.

$1.312, \quad 1.314,1.479,1.552,1.700,1.803,1.861,1.865,1.944,1.958,1.966,1.997, \quad 2.006$, $2.021,2.027,2.055,2.063,2.098,2.140,2.179,2.224,2.240,2.253,2.270, \quad 2.272, \quad 2.274$, $2.301, \quad 2.301,2.359, \quad 2.382,2.382,2.426,2.434,2.435,2.478,2.490,2.511,2.514, \quad 2.535$, $2.554,2.566,2.570,2.586,2.629,2.633,2.642,2.648,2.684,2.697,2.726, \quad 2.770, \quad 2.773$, $2.800, \quad 2.809, \quad 2.818, \quad 2.821,2.848, \quad 2.880, \quad 2.954,3.012, \quad 3.067,3.084,3.090,3.096,3.128$, $3.233, \quad 3.433, \quad 3.585, \quad 3.585$

Table 3 Fitted distributions and their abbreviations.

\begin{tabular}{lcc}
\hline Distribution & Abbreviation & References \\
\hline Topp-Leone OLL IEx & TLOLL-IEx & Proposed \\
IEx & IE & {$[7]$} \\
Exponential & E & {$[15]$} \\
Lindley & L & {$[9]$} \\
Generalized Lindley & GL & {$[9]$} \\
Generalized Gamma & GG & {$[9]$} \\
Exponential Power & EP & {$[15]$} \\
Power Quasi Lindley & PQL & {$[16]$} \\
Weibull & W & {$[17]$} \\
Sujatha & S & {$[10]$} \\
Generalized Sujatha & GS & {$[10]$} \\
\hline
\end{tabular}

Table 4 MLEs and the values of AIC, CAIC, and BIC statistics.

\begin{tabular}{lcccccc}
\hline Distribution & \multicolumn{3}{c}{ Estimated Parameters } & AIC & CAIC & BIC \\
\hline $\mathrm{TLOLL}(a, b, \lambda)$ & 6.4855 & 0.5668 & 2.0404 & 103.8239 & 104.1931 & 110.5262 \\
$\mathrm{IE}(\lambda)$ & 2.343 & & & 264.0296 & 264.0893 & 266.2637 \\
$\mathrm{E}(\beta)$ & 2.4513 & & & 263.7352 & 263.7949 & 265.9693 \\
$\mathrm{~L}(\theta)$ & 0.6545 & & & 240.3805 & 240.4402 & 242.6146 \\
$\mathrm{GL}(\theta, \alpha, \beta)^{*}$ & 9.3907 & 22.7198 & 4.771 & 106.0848 & 106.454 & 112.7871 \\
$\mathrm{GG}(\theta, \alpha, \beta)^{*}$ & 3.5861 & 2.6483 & 0.3044 & 103.9877 & 104.3569 & 110.69 \\
$\mathrm{EP}(\alpha, \beta)^{*}$ & 2.992 & 3.7061 & & 111.204 & 111.3858 & 115.6722 \\
& & & & & & (continued)
\end{tabular}


Table 4 MLEs and the values of AIC, CAIC, and BIC statistics. (Continued)

\begin{tabular}{lccccc}
\hline Distribution & \multicolumn{2}{c}{ Estimated Parameters } & AIC & CAIC & BIC \\
\hline $\mathrm{W}(\alpha, \lambda)^{*}$ & 3.843 & 0.088 & 275.8682 & 276.05 & 280.3364 \\
$\mathrm{~S}(\theta)^{*}$ & 0.0956 & & 243.5 & 243.63 & 244.93 \\
$\mathrm{GS}(\theta, \alpha)^{*}$ & 0.0972 & 14.473 & 244.54 & 244.68 & 243.97 \\
\hline
\end{tabular}

MLE, maximum likelihood estimation; AIC, Akaike information criterion; CAIC, consistent Akaike information criterion; BIC, Bayesian information criterion.

${ }^{\star}$ Estimated parameter values are obtained from related references.

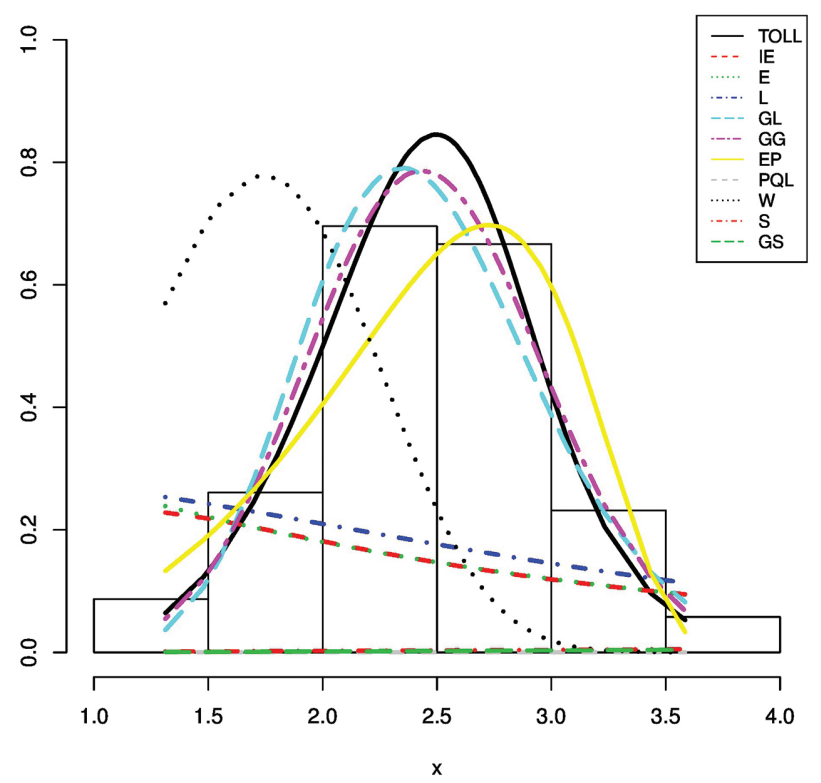

Figure 3 Graph for fitted distributions to the data set.

\section{CONFLICTS OF INTEREST}

The authors declare no potential conflict of interests.

\section{AUTHORS' CONTRIBUTIONS}

All authors contributes equally.

\section{ACKNOWLEDGMENTS}

We are very thankful to all referees for their precious time for the evaluation of the article. We also appreciate the correspondence of the editor.

\section{REFERENCES}

1. A. Keller, A. Kamath, U. Perera, Reliability Eng. 3 (1982), 449-473.

2. C. Lin, B. Duran, T. Lewis, Microelectronics Reliability. 29 (1989) , 619-626.

3. S. Dey, Data Sci. J. 6 (2007), 107-113.

4. G. Prakash, J. Modern Appl. Stat. Methods. 11 (2012), 16.

5. A. Abouammoh, A.M. Alshingiti, J. Stat. Comput. Simulation. 79 (2009), 1301-1315.

6. S. Nadarajah, S. Kotz, Interstat Electron. J. 14 (2003), 01-07.

7. I. Elbatal, Econ. Quality Control. 28 (2013), 125-133.

8. P. Oguntunde, O. Babatunde, A. Ogunmola, Int. J. Stat. Appl. 4 (2014), 113-116.

9. P. Oguntunde, A. Adejumo, J. Adv. Stat. Probability. 3 (2015), 1-7.

10. P. Oguntunde, A. Adejumo, O. Balogun, Appl. Math. 4 (2014), 47-55.

11. R. Bakoban, H.H. Abu-Zinadah, REVSTAT Stat. J. 15 (2017), 65-88. 
12. C.W. Topp, F.C. Leone, J. Am. Stat. Assoc. 50 (1955), 209-219.

13. E. Brito, G. Cordeiro, H. Yousof, M. Alizadeh, G. Silva, J. Stat. Comput. Simulation. 87 (2017), 3040-3058.

14. S. Chwastiak, J.B. Barr, R. Didchenko, Carbon. 17 (1979), 49-53.

15. N. Akdam, I. Kinaci, B. Saracoglu, Hacettepe J. Stat. 46 (2017), 239-253.

16. R.P. Covert, G.C. Philip, AIIE Trans. 5 (1973), 323-326.

17. S. Mondal, D. Kundu, Sankhya Ser. B. 81 (2019), 1-25. 